\title{
Comparative Study of Techniques used in the Generation Expansion Planning
}

\author{
F. Delgado, A. Ortiz, C. J. Renedo, F. Ortiz, M. Mañana \\ Department of Electrical and Energy Engineering \\ E.T.S.I.I., Cantabria University \\ Avenida de Castros, 39005 Santander (Spain) \\ Phone: +34-942-201376, Fax: +34-942-201385 \\ E-mail: delgadof@unican.es
}

\begin{abstract}
At the present time the generation expansion planning (GEP) has become a problem very difficult to solve for multiple reasons: many objectives, high uncertainties, very great planning horizon, etc. Its resolution by means of the exact traditional techniques, in numerous occasions, is not viable by the excessive time that is needed. For that reason, technical modern that allow the resolution in smaller time and with smaller accuracy in the solution are applied. Most of the approximate techniques are included inside a wider concept that is denominated Artificial Intelligence. In this article the more promising techniques of IA are studied indicating their applications in the PEG as well as their advantages and disadvantages
\end{abstract}

Keywords: Generation Expansion Planning, Optimization, Artificial Intelligence techniques.

\section{1.- Introduction}

The introduction of the electrical liberalized market established by Law 54/1997 of the Electrical Sector, the creation of the Iberian Market of the electricity between Spain and Portugal, the processes of fusion between electric companies or entrances of new agents will produce effects - negatives or positives- on the existing and future generating equipment. In this framework, the Generation Expansion Planning (GEP) acquires a crucial importance, mainly, for the investors.

The GEP is to determine What, When, Where y How generating equipment should connect to the system to satisfy the electrical demand fulfilling a series of restrictions - foreseen demand, reliability and safety in the supply ... with the purpose of satisfying one o more criteria - economic, of operation, environmental - in a long-term planning horizon [18], [21].

In the beginning of the planning, the minimization of the total costs (fixed and variables) was the only considered criterion. Later on, other criteria, security and quality of the supply, have been added. At the present time, a fourth criterion, the minimization of the polluting emissions has been added. Besides considering the previously mentioned criteria, a series of restrictions of the system must keep in mind. These can be countless depending on the exhaustive of the exposition of the problem: capacity constraints, production constraints, operation constraints...

To face this challenge - to consider so many aspects and so complex it is - the GEP mathematical modelling has been carried out, more or less trustworthily. This problem has been considered, traditionally, as a problem of optimization of large-scale size, non-linear, nonconvex, mixed-integer and stochastic. Their resolution has been carried out in multiple ways and applying different mathematical techniques with the purpose of finding, generally, the minimum cost of the generating system. At the present time, this problem has an added aspect: it is a multicriteria problem. This last aspect should be considered in the resolution of the problem

Depending on the considered planning horizon the problem of optimization -to obtain the best solution in the problem- of the GEP can be or not to be NP-hard. That is to say, it is not possible to guarantee finding the best possible solution in a reasonable time. As much as bigger is the planning horizon of the problem, major possibilities of being NP-hard.

Two types of techniques exist if it is considered the grade of accuracy of the obtained solution: exacts and approximates. It is guaranteed the optimum value of the problem with the exact techniques. In compensation, generally, the computer time is high and, in many occasions, inadmissible. With the second type of techniques - approximate techniques - the optimum value is not guaranteed. In compensation, a good solution is obtained at one time reasonable.

In following sections the different approximate techniques used in the PEG are detailed as well as their advantages and disadvantages. 


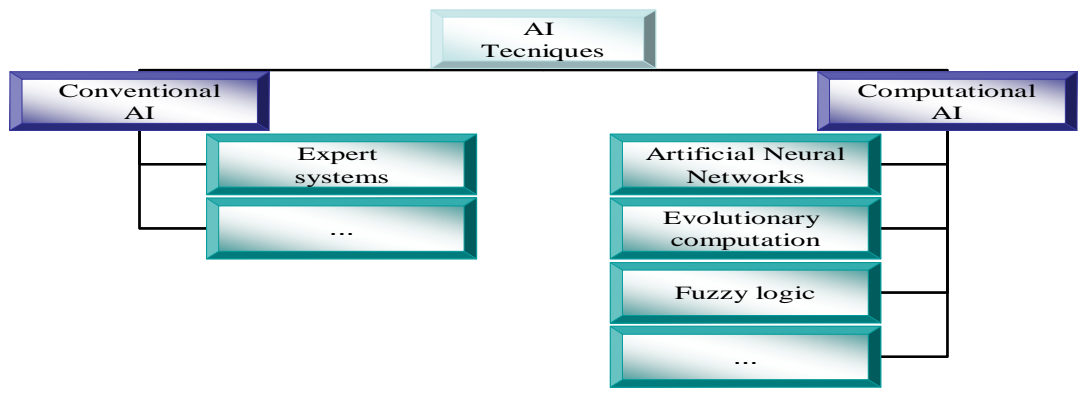

Scheme $\mathrm{n}^{\circ} 1$. AI techniques.

\section{2.-Artificial Intelligence (AI) techniques used in GEP}

\section{1.- Introduction}

Most of the approximate techniques are included inside a wider concept that is denominated Artificial Intelligence (AI). Many definitions exist of what AI means. These are related with the development of methods and algorithms that allow to the computers to behave in an intelligent way. The AI techniques can be classified according the scheme $\mathrm{n}^{\circ} 1$.

The methods that belong to the Conventional Artificial Intelligence (CAI) are characterized by the formalism and the statistical analysis. The rational activity is modeled by means of formal systems of rules and symbolic manipulation (logical systems). The Computational Artificial Intelligence (CoAI) implies development or iterative learning. The learning is based on empiric data.

Inside the CoAI a series of optimization techniques exists that could be classified according to scheme $\mathrm{n}^{\circ} 2$.

A heuristic is a technique that looks for good solutions (that is to say, almost optimum solution) at a reasonable computational cost, although without guaranteeing feasibility or optimal value. The classification of the heuristic techniques is shown in the scheme $\mathrm{n}^{\mathrm{o}} 2$.

The constructive heuristic techniques generate a solution adding components in an incremental way until completing the solution. They are, usually, determinist methods that offer the quickest resolution. In compensation, the obtained solutions usually are of very low quality to depend strongly on the problem that are solving. Habitually, in problems with many restrictions -it is our case - the obtained solutions are not feasible.

The local search heuristic techniques consist basically in an iterative process where a group of solutions is calculated starting from an only solution and a new one is chosen of the group. One of the simplest approaches to determine the new solution is to choose the best solution (evaluated with the objective function) of the neighborhood whenever it overcomes to the previous one (greedy approach). The algorithm stops when the current solution is not overcome. The last found solution is denominated local optimum solution. Nobody assures us that the local optimum solution will be the global optimums solution. This is the main disadvantage of these methods. Fundamental advantage of these techniques is the speed of resolution.

Finally, in many problems the exploration of all possible solutions is nonviable. For this reason, exploration strategies that simplify the search are applied.

The previous explanation justifies the non application of these two types of heuristic in the GEP.

A metaheuristic is an iterative process that guides a subordinated heuristic technique combining intelligently different concepts to explore and to exploit the search space, using learning strategies to structure the information with the objective of finding efficiently solutions near to the optimum one. These deterministic or stochastic methods have as purpose to avoid the fall in a local optimum solution in the search process. In the scheme $n^{\circ} 2$ a possible classification of these techniques is shown.

The operation of the metaheuristic techniques based on constructive methods consists on the 
initial application of the constructive methods to generate a solution. Next, this solution is improved with the local search methods. Also, the first ones are designed combining chance and evaluation. The introduction of the chance inside the procedure of optimization is due to the "myopia" -in each iteration the best option is always chosen without considering that this can take us later on to bad solutions - of these heuristic techniques. Introducing the chance in the selection of a new solution among a group of them is a way to avoid the mentioned problem.

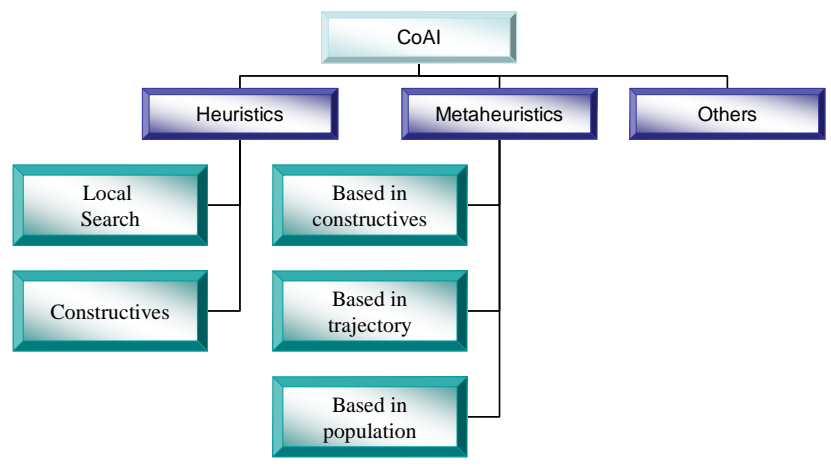

Scheme ${ }^{\circ}{ }^{2}$. CoAI techniques

Two algorithms of this type exist that are outstanding for their application in great quantity of problems (GRASP and Ant Colony method) but not in GEP.

The metaheuristic techniques based on trajectory has been developed modifying the methods of simple local search to escape from the local minima solutions. Two metaheuristic techniques of this type that have been applied in GEP exist: Taboo search and simulated annealing

Finally, the metaheuristic techniques based on population works with a group of solutions (population) in each iteration obtaining a new population. The obtained result depend on the population's treatment. In the scheme $n^{\circ} 3$ are shown the metaheurístic techniques of this type that are applied at the present time in the GEP.

Next, several AI techniques used in PEG are developed. These techniques have been used widely with interesting results. The techniques that are studied are inside the CoAI in the branches Others and Metaheurístics.

Other AI techniques exist (Expert systems, Taboo search, Simulated annealing, particle swarm,...) that have been applied directly in the PEG or like integral part of another technique. They are not developed due to his smaller importance in this field of study and by lack of space in the article.
The bases of the studied AI techniques are detailed in a brief way in the next sections. Also, those articles of special relevance that have applied the different AI techniques and their results are mentioned.

\section{2.-Computational Artificial Intelligence}

\subsection{1.-Others}

In the next sections the techniques of CoAI that are included inside the scheme $\mathrm{n}^{\mathbf{0}} 2$ in the branch Others are revised.

\section{Artificial Neuronal Networks (ANN)}

The human brain is a highly complex, no-linear and parallel system. It can carry out many operations simultaneously. This supposes an enormous power of calculation that allows the resolution of complex problems in a brief time in comparison with the present computers that process the information in a sequential way. The ANN try to reproduce these characteristics.

A neuronal network is a network with nodes neurons, by analogy with the human brain- that are interconnected through weighed connections (weights- $\mathrm{w}_{\mathrm{i}}$ ) like it is appreciated in the figure $\mathrm{n}^{\circ}$ 1. The weights are usually adjustable and can be trained through algorithms of learning and examples of training. 


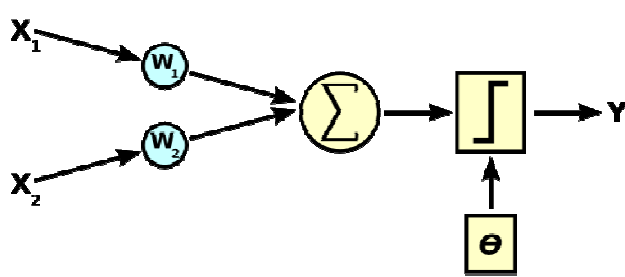

Figure $\mathrm{n}^{\circ}$ 1. Mathematical scheme of a neuron

The weights are multiplied by the entrances and they define with their value the relative importance of each one of them. The total entrance will be defined mathematically as:

$$
U_{n}=\sum_{i=1}^{n} w_{i} x_{i}
$$

The neuron will be activated if the total entrance overcomes a certain threshold $(\theta)$. The result will be defined by means of an activation function $(\varphi)$.These functions are nonlinear and vary between 0 and 1 . Mathematically it is written:

$$
Y=\varphi\left(U_{n}-\theta\right)
$$

The neurons of the network are grouped in layers and receive the same information that they process in an independent way (highly parallel system like the brain).

ANN are not programmed, are trained: adjustment of the weights that determines the degree of connection between the neurons of the network. For this reason, this technique is suitable to solve problems that have a difficult programming as they are the problems of GEP.

Finally, to point out that different types of nets exist: Kohonen, Hopfield, etc. In energy systems, most of the applications have been resolved with the neuronal network of Hopfield. [3].

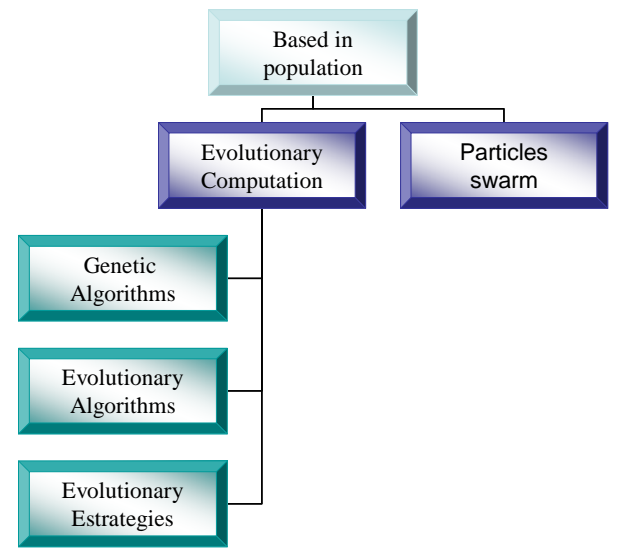

Scheme $\mathrm{n}^{\mathrm{o}} 3$. Metaheuristic techniques based on population

Sasaki Et al. [12], in 1991, formulated the GEP like an integer 0-1 programming problem. Next the problem was mapping using the Hopfield neuronal network that can handle a great number of restrictions by means of inequalities. Two systems were simulated: 10 generators during 5 periods of time and 20 generators during 10 periods. The objective was the minimization of the total costs fulfilling to a series of restrictions.

The result obtained with the neuronal network in the first test was compared with the result obtained with the exhaustive method Branch and bound. The error was a $6 \%$, approximately.

The exhaustive methods applied to the second test were not able to solve it due to the dimensions of the problem. However, the neuronal network was able to find solutions. In compensation, it was not guaranteed that the obtained minimum was optimal. On the other hand, the obtained minimum could not be assured that he was global optimum.

Zhaoguang $\mathrm{Hu}$, in 2002 [20], proposed a methodology of study of the GEP using IA techniques (ANN and fuzzy logic) as underlying tools that guided the study of diverse demand scenarios. These techniques allowed designing alternative roads of planning. The impact of the institutional strategies was also valued (economic, political and technological) on the electrification. Alternatives of planning were designed with these techniques. 
Fuzzy sets theory. Fuzzy logic

In the classic theory a set is a group of items. An item is or not in that set. In this new theory the object is not defined well within a set. It can be partial member of a set. For that reason, the sets are denominated fuzzy sets.

The property of the item to the set no longer will adopt discreet values $(0,1)$ but a value within the close interval $[0,1]$. The degree of property is defined by means of membership function to the studied fuzzy set: for each value that takes an entrance variable $x$ the membership function $\mu_{A}(x)$ provides the grade of property of this value to the fuzzy set $A$.

Therefore, the fuzzy logic allows managing vague information or information of difficult specification. The operation or the state of a system that it is not defined well can be controlled this way.

The systems based on fuzzy logic combine entrance variables, defined by means of "fuzzyficación" in terms of fuzzy sets, by means of groups of rules controlled by an inference motor that produce one or several fuzzy sets of outcomes. These last ones "are translated" to numerical values by means of an interface of “defuzzyficación” (figure ${ }^{\circ}$ 2).

The rules that govern the system can be learned with adaptative systems (they learn "observing" how people operate) or formulated by experts.

The most interesting characteristics of the fuzzy logic are its flexibility, its tolerance with the inaccuracy, its capacity to model no-linear problems and its base in the natural language.

It is very useful like integral part of an expert system. It will allow treating qualitative information or contradictory requirements.

In GEP, this technique is applied, generally, jointly with some other one (expert system, dynamic programming...) with different purposes (multiobjetive planning, consideration of uncertainties, consideration of qualitative requirements...).

In 1990, Satoh et al. [13] carried out an multiobjective planning combined linear programming and fuzzy logic. With this one last technique violables objectives and restrictions were designed.

In 1991, David et al. [2] carried out the GEP by means of dynamic programming. This program had a mechanism of decision making based on ES. The fuzzy logic was implemented inside the ES to formulate part of the procedure of decision making because some of the considerations were formulated in qualitative terms and it was necessary to consider contradictory requirements of the system.

In 1993, Hu Zhaoguang et al. [5] developed an ES with fuzzy rules that allowed carrying out an multiobjective planning of the GEP.

In 1994, Noor et al. [11] used this theory to consider the implicit uncertainty that the GEP possesses. By means of triangular fuzzy number they considered the existent uncertainties in the growth of the demand, discount percentage and escalation percentage. They evaluated several scenarios of planning by means of comparison among them.

In 2000, Ching-Tzong Su et al. [1] carried out the GEP by means of dynamic programming using the fuzzy logic to value the approaches of minimum cost -obtained by dynamic programming - and the derivative contamination of the calculated system jointly. As added advantage, the fuzzy logic allowed to eliminate routes and non feasible states. The computational limitations of the dynamic programming were reduced this way. A multiobjective optimization by means of the combination of an exact technique and another one of AI was carried out.

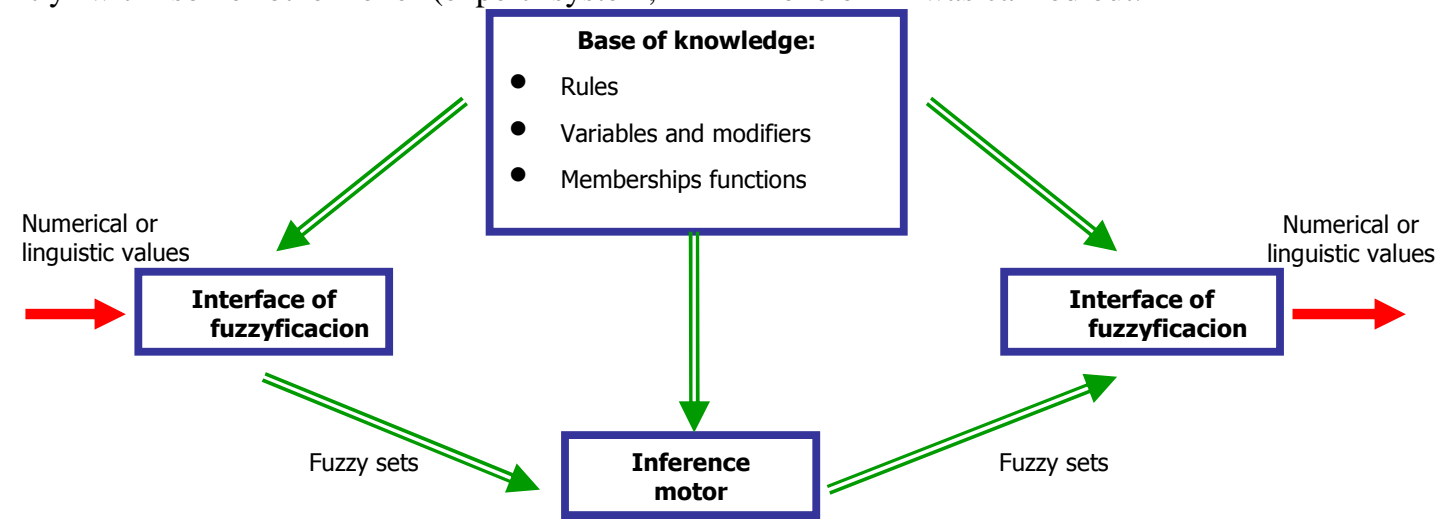

Figure $n^{\circ} 2$. Architecture of a fuzzy control system 


\subsection{2.- Metaheuristic techniques based on population}

In the next sections metaheuristic techniques based on population are developed that are inspired, generally, in biological processes. That is to say, the search processes of the solution are carried out using the biological processes like analogy. In addition, the algorithms of search work on a set of solutions avoiding with it the problem of the local minima solutions that have the metaheuristics based on trajectory.

\section{Evolutionary computation}

The evolutionary computation is a branch of the computer science that includes techniques that takes the natural evolution and genetics as analogy to solve search and a learning problems.

The natural evolution is based on the mechanisms of natural selection and sexual reproduction (crossover). In the nature, due to these mechanisms, the most capable individuals in a population are adapted better to the environment and they are the most successful for that reason. They have greater probabilities of surviving and of procreating a greater number of descendants. This implies that their genes (basic unit of codification of each one of the attributes of an alive being) will propagate in successive generations more and more numerous and better adapted to the environment by the systematic application of this mechanism.

This is a clear process of optimization: the better adapted individuals (better solutions) are taken, they cross (they mix) generating new individuals (new solutions) that will contain part of the genetic code (information) of their predecessors improving the average of the whole population's adaptation (better solutions that those of beginning).

This branch of AI includes, fundamentally, three techniques: genetic algorithms (GA), evolutionary programming and evolutionary strategies.

The GA applies the theory of the evolution better than the rest of evolutionary computational paradigms because it possesses the crossover, selection and mutation operators. The rest of paradigms doesn't have some of them. That is the reason for which it is the best technique of the three. As the above-mentioned consequence in this article is only developed the genetic algorithms.

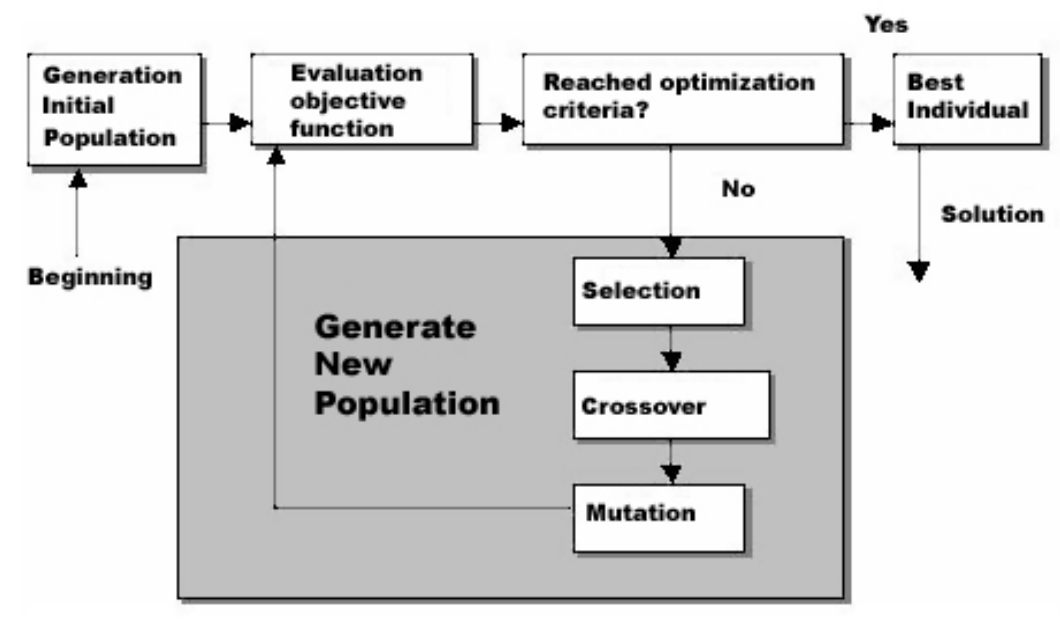

Figure $n^{\circ} 3$. Operation of GA

\section{Genetic algorithms (GA)}

The genetic algorithm of Holland. -creator of the technique in the decade of the 60 to solve problems of learning of the machines- gives bigger importance to the crossover operation that to the mutation operation.

The form of operation of the algorithm is shown in the figure $\mathrm{n}^{\circ} 3$.
In 1998, Y. M. Park et al. [19] proposed the resolution of the PEG by means of the combination of a refined genetic algorithm and dynamic programming. The second algorithm allows us to find the global optimum solution from the local optimum solution obtained by first algorithm. This way, it is overcome, simultaneously, the "curse of the dimensionality" and the problem of local optima solutions. It was applied successfully on two test 
systems with 15 plants, 5 types of generation and periods of planning of 14 and 24 years.

In 2000, Jong-Bae Park et al. [7] developed a genetic algorithm in which they incorporated a mechanism of stochastic crossover and a structure of the initial population's generation that it was applied on two cases of planning. One with a planning horizon of 14 year and the second with 24 years.

By means of dynamic programming the minimum cost was determined for the first test. For the second test, it was not possible their obtaining due to "the curse of the dimensionality" that suffers the dynamic programming.

Better solution was obtained - nearer to the real or the smallest solution- using the two mechanisms mentioned in the improved genetic algorithm with regard to the simple genetic algorithm and with regard to the tunnel-based dynamic programming also applied on the problem in the two cases.

Also, the improved genetic algorithm has run times smaller than times used in dynamic programming. However, it was slower with respect to the tunnel-based dynamic programming.

In 2004, T.S. Chung et al. [14] applied an improved GA to solve the GEP of a system of generation based on thermal power stations.

Numerous articles exist where the improved genetic algorithms are applied in the GEP [6], [4], [16]..

According to [8] the techniques based on AG that have been improved somehow (modification of the operators, hybridization with direct search techniques) produce the best results. In particular, the hybrid AG obtains, for the GEP of 6 years, a $90 \%$ of correct results, in the GEP of 14 years is the only technique that is able to obtain the correct result (12\%) and, in the PEG of 24 years, has the smaller error of all techniques.

This technique of AI is used jointly with other techniques of AI. In the articles [10] and [9] it has been used jointly with the simulated annealing technique, in the [15] with the Taboo search technique and in the [17] with particles swarm technique.
This technique, in optimization problems, falls less in local optimum solution than other techniques.

It is very simple of executing this algorithm in parallel architectures.

This technique can converge very slowly or not to converge in function of many parameters: the population's size, number of generations... To avoid these problems it is necessary that the search space or the population's size is defined in a certain range. To be possible the search spaces must be discreet or continuous of small range.

\section{3.-Conclusions}

\section{Artificial Neural Networks}

- The calculations made with this technique give important errors or he is not able to reach the solution.

-- With the purpose of overcoming the limitations that this technique possesses is used jointly with other techniques (fuzzy logic).

Fuzzy logic

- Very versatile technique used with many other exact and approximate techniques (dynamic programming, SE, linear programming, ..) to carry out complex plannings (multiobjetive, stochastic, with qualitative restrictions...)

-. The handling of qualitative restrictions - usual in the problems of PEG- is simple with this technique.

\section{Genetic algorithm}

- It is used in planning jointly with Taboo search, simulated annealing, dynamic programming, etc. This mixture improves the resolution of the problem: smaller times of resolution or better results.

- It is also applied directly with some improvement generating a bigger level of precision.

\section{Bibliography}

[1] Ching-Tzong Su; Guor-Rurng Lii; JiannJung Chen. Long-term generation expansion planning employing dynamic programming and fuzzy techniques. Proceedings of IEEE International Conference on Industrial 
Technology 2000 (IEEE Cat. No.00TH8482), 2000, pt. 2, 644-9 vol.2

[2] David, A. K.;Zhao, Rongda. An expert system with fuzzy sets for optimal planning. IEEE Transactions on Power Systems, v 6, n 2, Feb, 1991, p 59-65

[3] El-Hawary, M.E. Applications of artificial neural networks in electric power systems operational planning. International Journal of Engineering Intelligent Systems for Electrical Engineering and Communications, v 7, n 1, Mar, 1999, p 49-61

[4] He Feng; Xiong Xin-yin; Wu Yao-wu. Generation expansion planning of power system by partheno-genetic algorithm. Relay, v 31, n 6, June 2003, 26-34

[5] Hu Zhaoguang; Wang Pingyang; Zhou Xiaoxin; $\mathrm{Hu}$ Zhaoyi. Fuzzy decision support system for generation expansion planning with multiobjective functions. Power System Technology, n 2, March 1993, 38-43

[6] Jong-Bae Park, Jin-Ho Kim, Kwang Y. Lee. Generation Expansion Planning in a Competitive Environment Using a Genetic Algorithm. IEEE TRANSACTIONS ON POWER SYSTEMS, VOL. 15, NO. 3, AUGUST 2000.

[7] Jong-Bae Park; Young-Moon Park; JongRyul Won; Lee, K.Y. An improved genetic algorithm for generation expansion planning. IEEE Transactions on Power Systems, v 15, n 3, Aug. 2000, 916-22

[8] Kannan, S. Slochanal, S.M.R. Padhy, N.P. Application and comparison of metaheuristic techniques to generation expansion planning problem. Power Systems, IEEE Transactions on. Feb. 2005. Volume: 20, Issue: 1, pages 466475

[9] Mehmet Yildirim and Kadir Erkan. Determination of acceptable operating cost level of nuclear energy for Turkey's power system. Energy, Volume 32, Issue 2, February 2007, Pages 128-136

[10] Mehmet Yildirim; n,y, Kadir Erkan; and Semra Ozturk. Power generation expansion planning with adaptive simulated annealing genetic algorithm. Int. J. Energy Res. 2006; 30:1188-1199

[11] Noor, S.F. ; McDonald, J.R. Using fuzzy numbers in generation expansion planning. ISAP '94. International Conference on Intelligent System Application to Power Systems, 1994, pt. 1, 145-51 vol.1

[12] Sasaki, H.; Kubokawa, j. A solution of generation expansion planning problem by means of neural network. Proceedings First International Forum on Applications of Neural Network to Power Systems, Seattle, 1991

[13] Satoh, H.; Serizawa, Y. Fuzzy decisionmaking on electric energy strategy for long- term generation expansion planning. Power Systems and Power Plant Control 1989. Selected Papers from the IFAC Symposium, 1990, 23741

[14] T. S. Chung, Y. Z. Li and Z. Y. Wang. Optimal generation expansion planning via improved genetic algorithm approach. International Journal of Electrical Power \& Energy Systems, Volume 26, Issue 8, October 2004, Pages 655-659

[15] Tung-Sheng Zhan ; Ming-Tong Tsay; Sung-Ling Chen. An improved genetic algorithm for utility generation expansion planning in a competitive market. Engineering Intelligent Systems for Electrical Engineering and Communications, v 12, n 3, Sept. 2004, 167-73

[16] Wu Yaowu; Hou Yunhe; Xiong Xinyin; Lu Lijuan. A model for generation expansion planning of power system based on genetic algorithm. Power System Technology, v 23, n 3, March 1999, 10-14

[17] Wu Yao-wu; Wang Zheng; Tang Quan; Xiong Xin-yin; Lou Su-hua. Generation expansion planning of hydro-thermal mixed power system based C-PSO. Relay, v 34, n 9, 1 May 2006, 64-9

[18] Wu, Felix ; Yen, Zheng; Hou, Yunhe; Ni, Yixin. Applications of AI techniques to generation planning and investment. 2004 IEEE Power Engineering Society General Meeting, v 1, 2004 IEEE Power Engineering Society General Meeting, 2004, p 936-940

[19] Y. M. Park, J. B. Park and J. R. Won. A hybrid genetic algorithm/dynamic programming approach to optimal long-term generation expansion planning. International Journal of Electrical Power \& Energy Systems, Volume 20, Issue 4, May 1998, Pages 295-303

[20] Zhaoguang Hu. Study on methodology of generation expansion planning for power restructuring. PowerCon 2002. 2002 International Conference on Power System Technology Proceedings (Cat. No.02EX572), 2002, pt. 1, 388-92 vol.1

[21] Zhu, Jinxiang, Chow, Mo-yuen. Review of emerging techniques on generation expansion planning. IEEE Transactions on Power Systems, v 12, n 4, Nov, 1997, p 1722-1728 